\title{
Flower Jelly Printer: Slit Injection Printing for Parametrically Designed Flower Jelly
}

\author{
Mako Miyatake \\ The University of Tokyo \\ mako-miyatake@g.ecc.u-tokyo.ac.jp \\ Yuji Sekiya \\ The University of Tokyo \\ sekiya@nc.u-tokyo.ac.jp
}

\author{
Koya Narumi \\ The University of Tokyo \\ narumi@akg.t.u-tokyo.ac.jp \\ Yoshihiro Kawahara \\ The University of Tokyo \\ kawahara@akg.t.u-tokyo.ac.jp
}

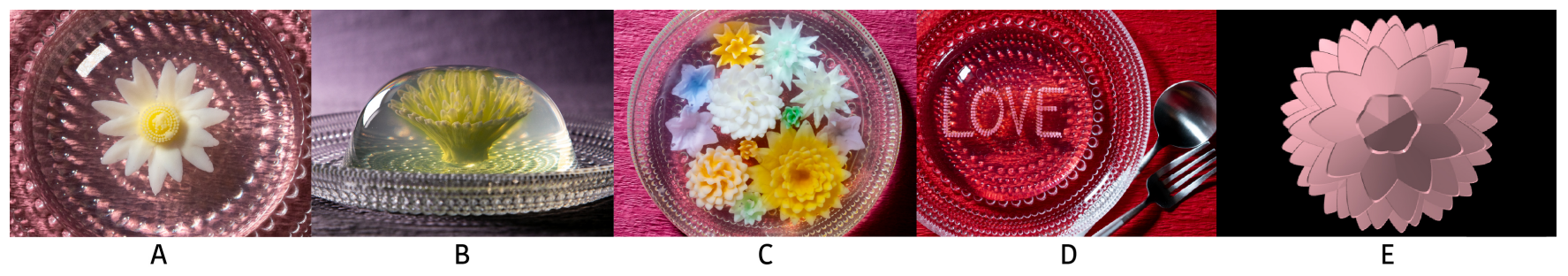

Figure 1: Flower jellies made with Flower Jelly Printer. (A) Marguerite, (B) Mum, (C) A cake with several types of flowers, (D) A printed message, and (E) A schematic preview of dahlia in the computational design tool.

\begin{abstract}
Flower jellies, a delicate dessert in which a flower-shaped jelly floats inside another clear jelly, fascinate people with both their beauty and elaborate construction. In efforts to simplify the challenging fabrication and enrich the design space of this dessert, we present Flower Jelly Printer: a printing device and design software for digitally fabricating flower jellies. Our design software lets users play with parameters and preview the resulting forms until achieving their desired shapes. We also developed slit injection printing that directly injects colored jelly into a base jelly, and shared several design examples to show the breadth of design possibilities. Finally, the user study with novice and experienced users demonstrates that our system benefits creators of all experience levels by iterative design and precise fabrication. We hope to enable more people to design and create their own flower jellies while expanding access and the design space for digitally fabricated foods.
\end{abstract}

\section{CCS CONCEPTS}

- Human-centered computing $\rightarrow$ User interface toolkits; • Applied computing $\rightarrow$ Computer-aided manufacturing.

This work is licensed under a Creative Commons Attribution-Share Alike International 4.0 License.

CHI '21, May 8-13, 2021, Yokohama, Japan

(c) 2021 Copyright held by the owner/author(s).

ACM ISBN 978-1-4503-8096-6/21/05.

https://doi.org/10.1145/3411764.3445346

\section{KEYWORDS}

Digital food fabrication; food 3D printer; fabrication techniques; interactive food; food-interaction design.

\section{ACM Reference Format:}

Mako Miyatake, Koya Narumi, Yuji Sekiya, and Yoshihiro Kawahara. 2021. Flower Jelly Printer: Slit Injection Printing for Parametrically Designed Flower Jelly. In CHI Conference on Human Factors in Computing Systems (CHI '21), May 8-13, 2021, Yokohama, Japan. ACM, New York, NY, USA, 10 pages. https://doi.org/10.1145/3411764.3445346

\section{INTRODUCTION}

Flower jelly, as illustrated in Figure 1, is an artistic dessert in which an intricate flower-shaped jelly floats inside a clear jelly matrix. Appearing just like real flowers suspended in glass, these handmade creations demonstrate patissiers' extremely skilled craftsmanship. In the manual process, they need to inject the knife from the top side of the jelly. But the final shape is always upside-down, so they always have to imagine the finished shape of the opposite side. Also, they must care about controlling the slight angles of the knife with equal and appropriate distances to finally make the flower with the designed balance and numbers of petals. In general, many of the cooking process of well-prepared dishes and desserts involve complicated manual labor. Thus we implemented a 3D preview to visualize the final shape and modified a $3 \mathrm{D}$ printer to achieve precise motions. Our motivation is to maximize the aesthetic possibilities and simplify the fabrication of this soft, fragile, and difficult to produce delicacy by means of digital fabrication techniques.

In the typical cooking process, patissiers commonly suffer from the following issues in the manual procedure: (1) Precise and accurate control of the hand while working with the soft material; (2) Prolonged concentration and effort required by the slow, manual 
process; and (3) Inability to undo or redo mistaken cuts and fills.

We believe that digital fabrication techniques are key to solving these problems. In general, introducing digital fabrication tools and processes into traditional manual cooking processes enables:

1. Precise fabrication of both complicated organic shapes and non-organic mathematical geometries

2. Less human labor required to produce the designed structure due to automation

3. Trial-and-error design iteration in software, allowing users to experiment until they find their desired structures

Although digital fabrication tools have been already introduced into many food fabrication processes, they cannot be applied to all types of food production. Unlike chocolates which solidify immediately at room temperature when extruded from a heated nozzle, hydrogel-based materials (e.g., gelatin and agar) take a long time to coagulate and retain the form, resulting in deformation while printing. Therefore, it is challenging to $3 \mathrm{D}$ print flower jellies using existing additive 3D food printing methods.

Instead, we present Flower Jelly Printer, a novel printing platform that not only addresses the challenges of manual fabrication procedures but also extends the design space of flower jellies. Our computational design tool allows users to parametrically play with the shape of the flower jelly and generates 3D previews until they find the exact parameters of their favorite designs. We overcome material limitations of existing food printers by developing a slit injection printing technique that directly injects colored jelly into a base jelly. Furthermore, we examined the material properties of jellies, introduced mechanical designs into the proposing printer, and built path planning algorithms to establish the digital fabrication of flower jellies.

We demonstrate a variety of design patterns ranging from a simple flower made of few petals to a complex flower with floral stamens in addition to multiple layers of petals. We also fabricated challenging non-floral geometric designs that require precise motions to create. Furthermore, to clarify how our proposed techniques solve the above-mentioned issues patissiers currently face, we recruited both novice and experienced flower jelly creators, and conducted a user study. From the user study, we found that even novice users could easily make flower jellies and professionals could test different patterns until they were satisfied with their design.

The main contributions of this paper are as follows:

- Presenting a computational design tool to digitally create and preview the shape and structure of flower jelly

- Developing a slit injection printing technique that can directly inject colored jelly into a base jelly

- Optimizing materials and methods of hydrogel-based 3D printing using two materials for colored jelly and base jelly

- Producing example flower jelly artifacts that demonstrate a variety of design and fabrication possibilities enabled by our approach

- Conducting a user study for novice and experienced participants to examine the effect of our system

\section{RELATED WORK}

\section{Digital Food Fabrication}

Recently, digital fabrication techniques have been introduced into food fabrication processes, represented by the fact that food 3D printers are increasingly available on market $[1,3,11,17]$. This growing availability has promoted interest in research on digital food fabrication [16, 18]. For example, Digital Gastronomy leverages digital fabrication tools to create novel recipes such as $3 \mathrm{D}$ printed noodles and a CNC milled sweet potato [12,19-21].

Especially, extrusion-based additive manufacturing is frequently utilized in human-computer interaction (HCI) to achieve unique experiences with food. FoodFab [10] is the system that creates perceptual illusions with a food 3D printer by changing infill patterns and infill densities. Transformative Appetite [15] extrudes edible materials (e.g., gelatin, cellulose) to fabricate shape-changing edible films that transform into 3D shapes through water adsorption. As another example, EdiPulse [7] extrudes cheerful messages and activity logs with a chocolate $3 \mathrm{D}$ printer to enrich the exercise experience. Although these prior works have spread the design space of current digital food fabrication, their relatively simple extrusion processes are not suitable for the fabrication of flower jelly, which needs to build a delicate and tall structure with slowly curing and deformable materials like gelatin.

There are also several prior works on digital food fabrication that leverage subtractive manufacturing processes. This category includes CNC milling [12], stamping and grooving with digitally controlled jigs $[9,14]$ or engraving with a laser cutter [2]. However, these subtractive fabrications are not very suitable for the soft and therefore fragile nature of jelly.

This paper aims at developing a novel digital fabrication technique to widen the design freedom of current flower jellies by design iteration using $\mathrm{CAD}$ software and the precise and automated fabrication enabled by our printer.

\section{D Printing in the Granular Gel Support}

Although they are not related to food, there are a few prior works on 3D printing materials in a gel support, which are similar to our approach. In the field of material science, Hinton et al. developed the technique to 3D print protein and polysaccharide hydrogels [6] and PDMS elastomer [5] for biological and medical applications. They used microparticle gel materials ground in a mixer or a centrifuge as a supporting bath to sustain the printing inks until they are fully cured. Likewise, Rapid Liquid Printing $[4,8]$ utilized the granular gels as the supporting bath to 3D print large objects in any direction with faster speed.

These previous studies used the firm jelly crushed into a granular structure. Thus the finished structure with the jelly support is like porridge, so it is impossible to eat them as a firm jelly. On the other hand, our proposing method prints jelly ink into the "bulk" (i.e., not granular) firm jelly, so we can take it out from the mold and eat the finished jelly right after it is printed while sustaining the predesigned aesthetic shape. This new process was needed to keep the outer shell transparent enough to clearly see the inner flower and to make the flower jelly aesthetically authentic. To achieve this goal, we developed slit injection printing. 


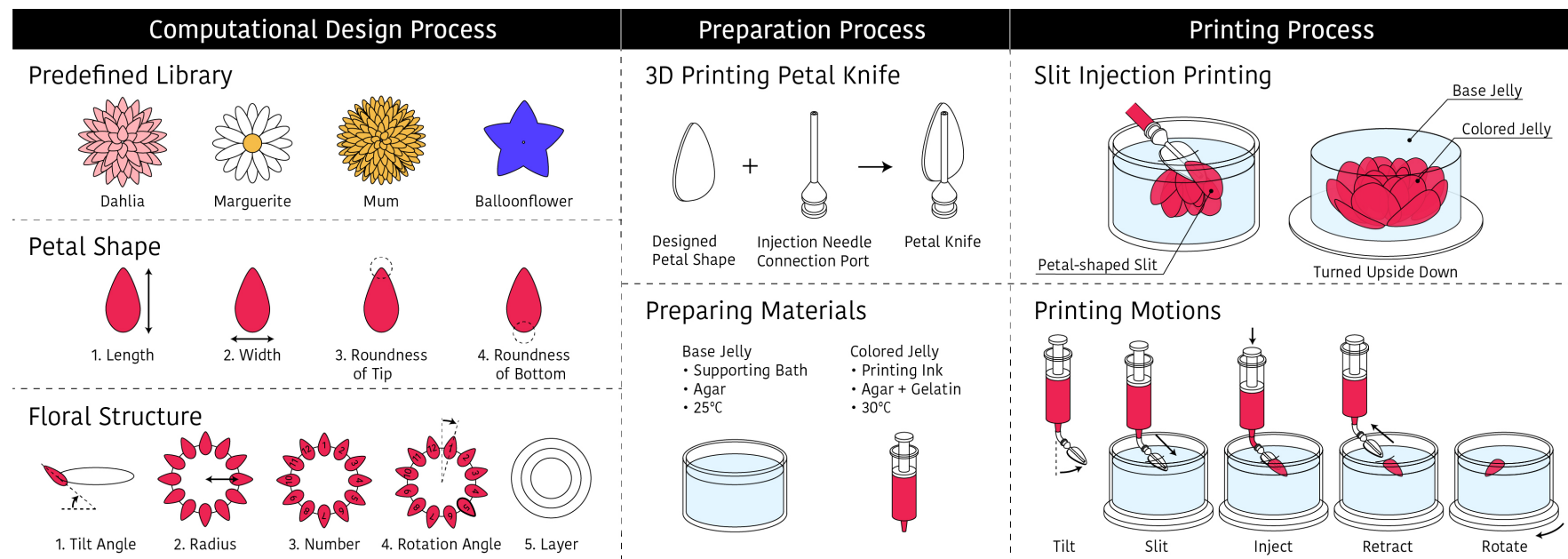

Figure 2: Overview of the proposed system. In the computational design process, our design software allows users to create their favorite flower jellies by changing parameters. In the preparation process, users prepare injection knives and printing materials before printing the desired jelly. In the printing process, the proposed slit injection printing completes the fabrication of flower jellies inside the base jelly structure.

We note that we found existing work that takes a similar approach to ours, which injects grains of food (e.g., silver dragées) inside a firm jelly [13]. However, this suffers from building 3D structure and ended up with 2D patterns. Also, this approach needed to fill the injection holes on the surface of the jelly with uncured jelly after the print, while our approach does not need such a postprocess. We will describe our method from the next section.

\section{FLOWER JELLY PRINTER}

\subsection{Overview}

Flower Jelly Printer is a printing platform that allows users to design flower jellies and fabricate them precisely through digital design and fabrication. Figure 2 gives an overview of our system: (1) Users design their desired flower jelly with our computational design tool; (2) Users fabricate custom tool attachments for the printer and prepare two jelly materials; (3) Our 3D printer prints the designed flower jelly by slit injection printing. In this section, we will follow each step in detail.

\section{Expected Target User and Usage}

Flower jellies are made by people who enjoy cooking and making creative meals on a special day. Also, a few restaurants hire experts to serve this dessert. Thus our target users are both novice cooking lovers and chefs. We think our printer is not useful for everyone but for those who love cooking using smart devices like blenders, slow cookers, and so on. For those people, trial and error iteration using software is helpful, because they want to achieve their own design without long-term practices. For chefs, our printer saves their time and effort to make delicate desserts. While printing, they can do other jobs. Reliably producing the same beautiful desserts without hiring an expert chef is one of the biggest goals for professionals.

\subsection{Computational Design Process}

To support the trial-and-error design iteration, we built a parametric design interface with Rhinoceros and Grasshopper. As shown in Figure 3, we provided a predefined library of example flower forms to suggest default parameters. Otherwise, novice users may find it overwhelming to set all the parameters of the flower jelly design from scratch. After selecting their favorite type of flower from the library, users launch the software of the chosen type to customize it. Currently, this library contains 6 types of flowers: dahlia, balloon flower, mum, allium roseum, marguerite, and sunflower.

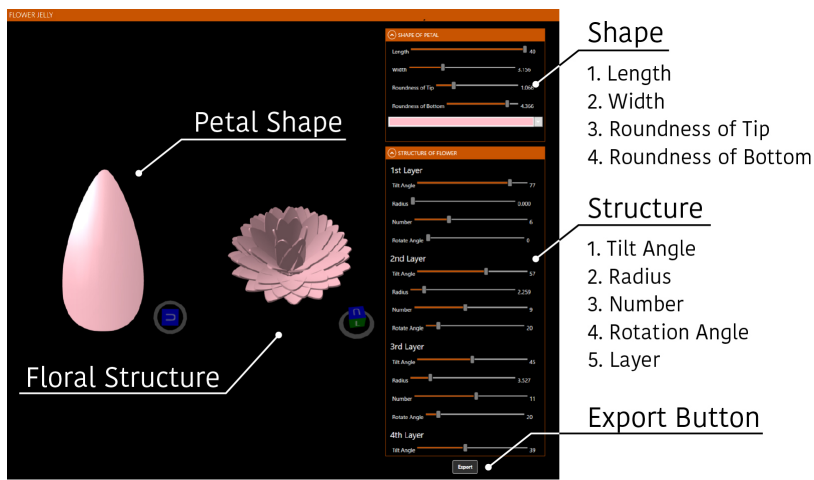

Figure 3: Design interface. The 3D preview of floral petal (left). The 3D preview of whole flower (center). Users can tune parameters for the shape of each petal (top right) and the whole appearance (bottom right), and iterate the design by checking the visualized model.

Next, users can design the shape of each petal (Figure 3 left). Our software offers several predefined shapes with customizable design 
parameters to achieve a wide variety of shapes; from sharp, narrow petals like marguerites to rounded, broad ones like roses. Users can specify petal shapes by setting 4 parameters: (1) length, (2) width, (3) roundness of the tip, (4) roundness of the bottom by dragging sliders to change design parameters.

Users can also customize the overall structure of the flower (Figure 3 center). All floral components have the same axis of rotation of each flower and every group of petals are radial symmetrically arranged in each layer. Thus, users can define most flowers by 4 parameters of each layer: (1) tilt angle of petals, (2) number of petals, (3) radius of the layer, and (4) rotation angle of petals. Users can also select the total number of petal layers.

Through the designing process, a 3D preview corresponding to the current set of parameters gives users a quick way to visualize the final result of their design. Users can change the structure dynamically until they find the ideal output, allowing every flower to be different and special. Once users are satisfied with the design, they can export an STL file for making the petal knife and a custom G-code file for the actual printing process of flower jellies.

\subsection{Preparation Process}

Before printing, users need to fabricate a custom petal knife and prepare jellies as printing materials.

The petal knife can be 3D printed from our design software. Once the design of the floral petal is finalized, our software generates an STL file of the petal knife that combines the designed petal shape, an injection needle (inner diameter: $0.4 \mathrm{~mm}$ ), and a connection mechanism to a silicone tube. The petal knife works not only as a knife to make a slit but also as a needle to inject colored jelly into the base jelly. In this study, we printed petal knives using a 3D printer Objet 260 Connex3 with a biocompatible material MED610 (Figure 4).
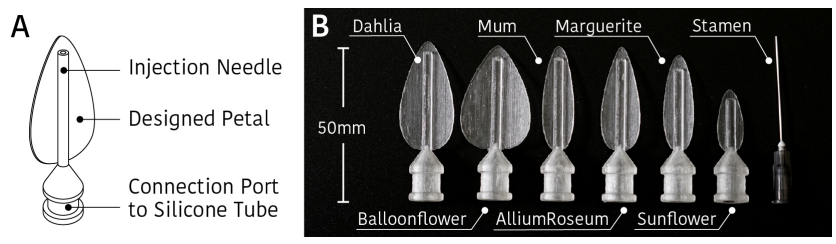

Figure 4: Petal knives. (A) A single petal knife is composed of an injection needle, a designed petal, and a connection port to silicone tube. (B) Various types of $3 \mathrm{D}$ printed petal knives.

To 3D print flower jelly, we prepared two different print materials: (1) base jelly, and (2) colored jelly.

For base jelly, we used Pearl Agar, flavored water, and sugar and prepared the ingredients as follows: Pour 1 l of flavored water into a saucepan and bring to a boil. Once boiling, add $48 \mathrm{~g}$ of agar and $136 \mathrm{~g}$ of sugar and stir until dissolved. Once fully dissolved, ladle equal amounts into each glass container. Transfer to the fridge to set for $2 \mathrm{~h}$.
For colored jelly, we used feleaf fellyace granular gelatin, milk, agar, sugar, and food coloring and prepared in the following method: Pour $400 \mathrm{ml}$ of milk into a saucepan and bring to a boil. Once boiling, heat gently over medium heat, add $6 \mathrm{~g}$ of agar and $100 \mathrm{~g}$ of sugar and stir until dissolved. Once fully dissolved, add $12 \mathrm{~g}$ of gelatin and stir until dissolved. Pour into a glass container, add the food coloring and stir until dissolved. Transfer to a water bath at $30{ }^{\circ} \mathrm{C}$.

\section{Viscosity of Colored Jelly}

Because the viscosity of the colored jelly has a large effect on the final look and finish of the petals, we experimentally determined the relationship between viscosity and gel temperature (shown in Figure 5). Results clarified that $30{ }^{\circ} \mathrm{C}$ is the optimal temperature of the colored jelly. Figure 5 (B) also shows how low viscosity in the colored jelly results in a lack of uniformity because the base jelly can push out the colored jelly, leaving gaps.
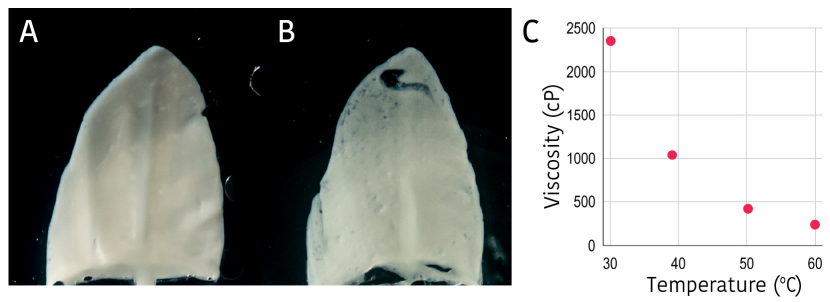

Figure 5: Viscosity of colored jelly. (A) No gaps in the final look of the petal at $30{ }^{\circ} \mathrm{C}$. (B) A lack of uniformity at $60^{\circ} \mathrm{C}$. (C) The graph shows the relationship between viscosity and temperature of colored jelly.

\subsection{Printing Process}

We propose a novel slit injection printing technique to directly inject colored jelly into a base jelly. Custom-shaped cutting and injection knives allow for the printing of a variety of shapes while a motorized turntable base enables easy printing of rotationally symmetric forms. Details about the proposed printer and its printing paths are described in more detail below.

\section{Slit Injection Jelly Printer}

To implement the slit injection printing technique, we modified a 3D printer Ender 3 by adding a motorized turntable and a DIY paste extruder with a petal knife. This printer is compatible with the G-code toolpath compiled by our design software. Below is the hardware setup of our printer:

1. Paste Extruder We designed a custom paste extruder to change the angle of the tip during the slitting process (Figure 6). We use a linear rail and a feed screw to convert the rotation of the motor (Nema 17 stepper motor) into the linear movement which pushes the plunger of the syringe (TELMO Syringe $30 \mathrm{ml}$ ). To enable changing the angle of the petal knife, we use a silicone tube (Leobro silicone tube $4 \mathrm{~mm} \times 6 \mathrm{~mm}$ ), which allows colored jelly to flow from the syringe to the petal knife regardless of knife angle. An MG90S servo motor mounted between the syringe and petal knife enables the knife to tilt to the desired angle. 


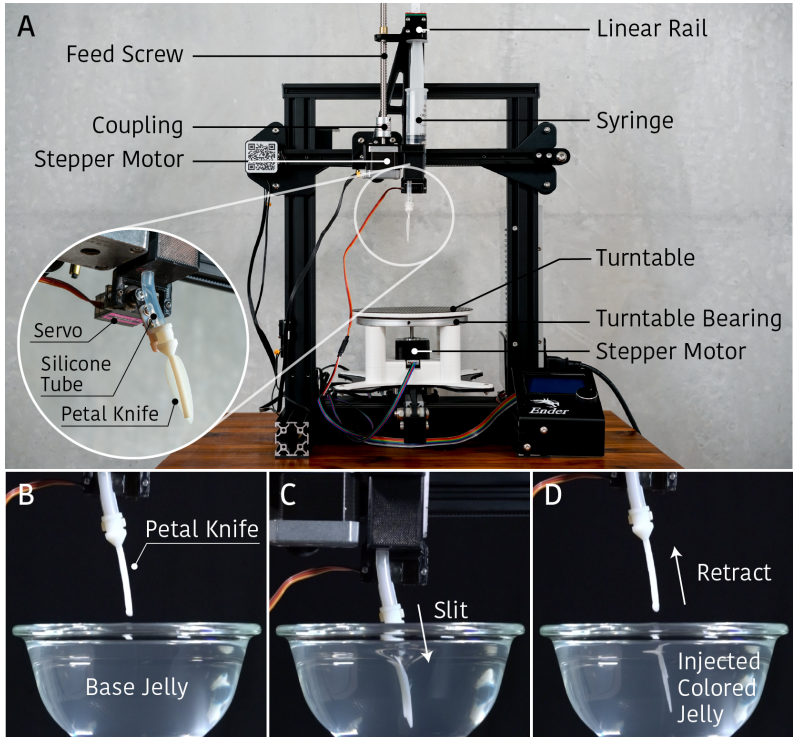

Figure 6: (A) The overview of the proposed printer. (B-D) The slit injection process. First, the petal knife makes a slit inside a base jelly. Then the printer retracts the knife while injecting colored jelly.

2. Motorized Turntable We replaced the standard printer's heating bed with our DIY motorized turntable. We use a Nema 17 stepper motor controlled by a BigTreeTech SKR v1.4 mainboard to control the rotation of the turntable. On the surface of the table, we put a nonslip sheet to prevent the container from slipping or falling off the table.

\section{Path Planning Algorithm}

Our software converts the parameters of the designed floral structure into the printing path, written as G-code. In order to make the finished flower jelly more aesthetically pleasing, printing paths are generated as shown below:

1. Tilt Angle of the Knife Tilt angles of the cutting knife are converted into the desired angle of the servo motor and then set using the M280 command of G-code via the mainboard.

2. Starting Position The knife's starting position $\left(x_{\text {start }}, z_{\text {start }}\right)$ corresponds to the position of the bottom of the petal (Figure 7). The coordinates are calculated by radius $(r)$, tilt angle $\left(\theta_{\text {tilt }}\right)$, tube length $\left(l_{\text {tube }}\right)$, and petal length $\left(l_{\text {petal }}\right)$ using the following equation:

$$
\left(\begin{array}{c}
x_{\text {start }} \\
z_{\text {start }}
\end{array}\right)=\left(\begin{array}{c}
x_{0}+r-\left(l_{\text {tube }}+l_{\text {petal }}\right) \sin \theta_{\text {tilt }} \\
z_{0}-\left(l_{\text {tube }}+l_{\text {petal }}\right)\left(1-\cos \theta_{\text {tilt }}\right)
\end{array}\right)
$$

We note that the initial cordinate of the knife $\left(x_{0}, z_{0}\right)$ are given by the user's calibration before printing.

3. Knife Insertion The path of knife insertion is determined by tilt angle and petal length. This motion is operated by relative coordinates $\left(\Delta x_{\text {insert }}, \Delta z_{\text {insert }}\right)$ from the coordinates of the starting position (Figure 7).

$$
\left(\Delta x_{\text {insert }}, \Delta z_{\text {insert }}\right)=\left(l_{\text {petal }} \sin \theta_{\text {tilt }},-l_{\text {petal }} \cos \theta_{\text {tilt }}\right)
$$

4. Injection of Colored Jelly \& Retract Knife While pulling the petal knife out from the base jelly, the printer pushes the syringe plunger to inject colored jelly. The length of extrusion $\left(l_{\text {length }}\right)$ is converted from the volume of the petal $\left(V_{\text {petal }}\right)$ by the following equation. $a$ is the ratio of the length of the syringe to flow of the colored jelly, which differs depending on the printer setup. We multiply the converted value by 1.2 because a small amount of injected colored jelly is extracted while retracting the petal knife from the base jelly.

$$
l_{\text {extrusion }}(\mathrm{mm})=V_{\text {petal }}\left(\mathrm{mm}^{3}\right) \cdot \frac{1}{1000}\left(\mathrm{~mL} / \mathrm{mm}^{3}\right) \cdot a(\mathrm{~mm} / \mathrm{mL}) \cdot 1.2
$$

5. Rotation of the Bowl The rotation is executed (1) before, (2) during, and (3) after each layer printing process. (1) Users set the rotation angle $\left(\theta_{\text {rotate }}\right)$ to prevent petals in a specific layer from overlapping petals on another layer. The motorized turntable rotates the desired angle before printing each layer. (2) Users set the number of petals $(n)$ in each layer. These petals are arranged in radial symmetry, the angle between petals are calculated by the quotient of $360^{\circ}$ and petal number. After each petal is printed by inserting motion, the turntable rotates the calculated angle $\left(\frac{360^{\circ}}{n}\right)$ for the next petal. (3) Once a group of petals on each layer are printed, the turntable stops at the rotation angle $\left(\theta_{\text {rotate }}\right)$. To set it to the initial position, the turntable rotates back the angle $\left(-\theta_{\text {rotate }}\right)$.
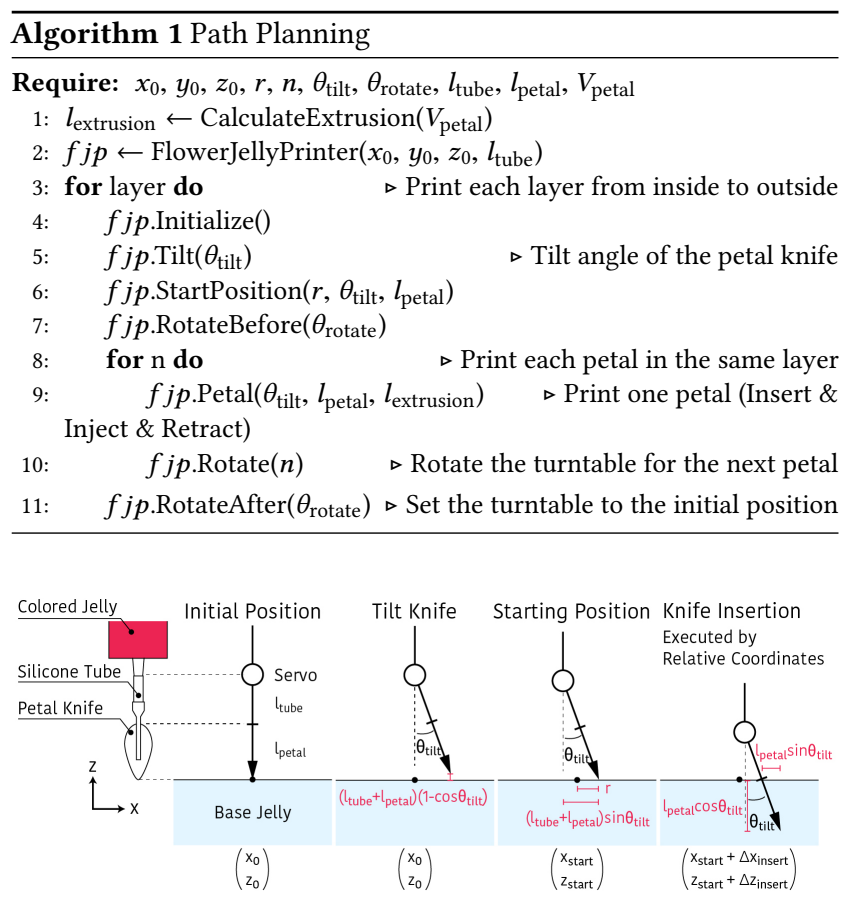

Figure 7: Printing motions. 


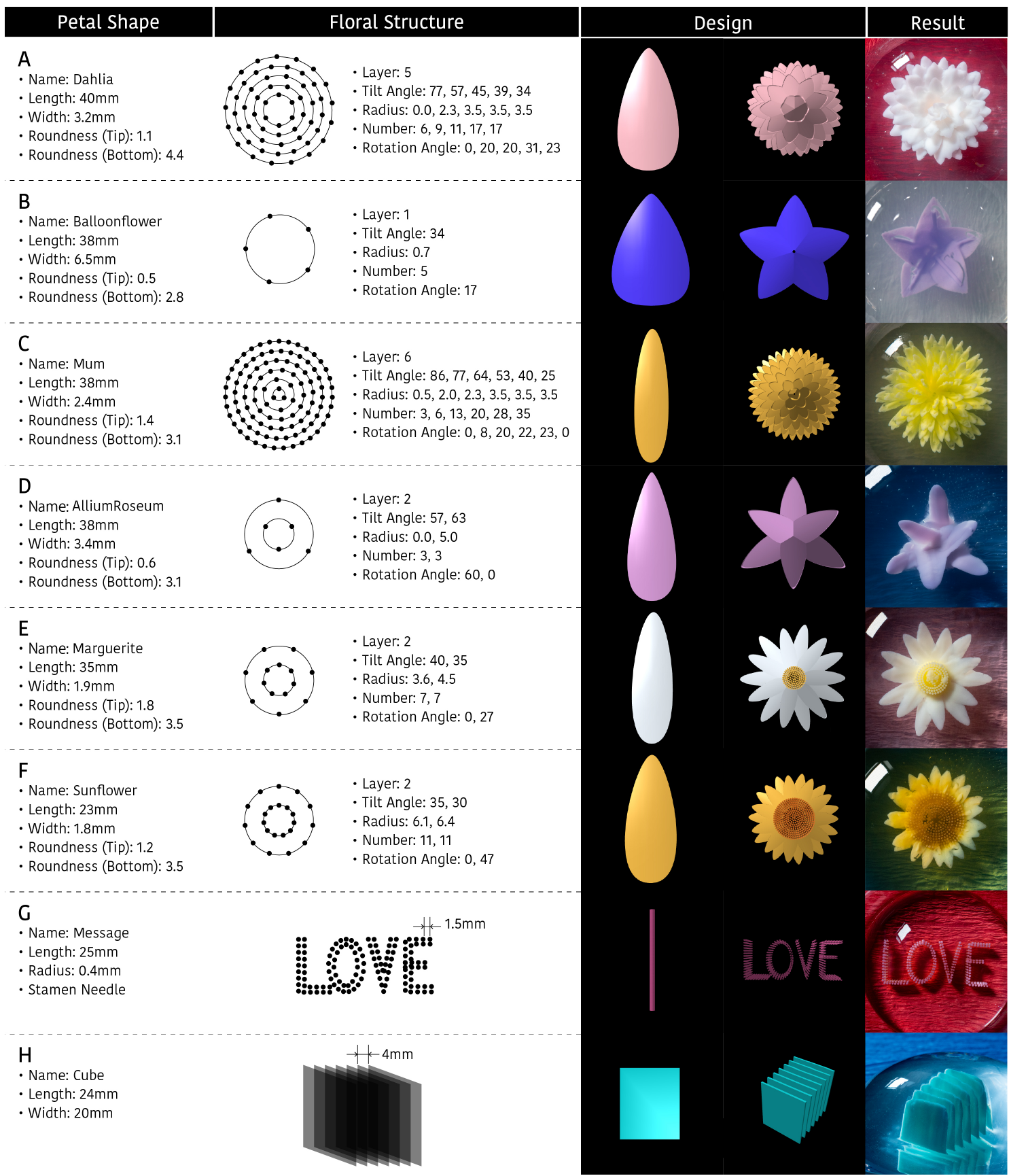

Figure 8: Demonstrations of several printed patterns. The columns show the basic information for the petal, the whole structure of the printed objects, the schematic 3D images of the petal and the structure, and the photographs of printed objects, respectively.

\section{DEMONSTRATIONS}

Using our predefined library, users can create various kinds of flower jellies. Figure 8 shows results made using the default parameters of the library. The printing time of a single flower depends on the number of petals: from $5 \mathrm{~min}$ to $30 \mathrm{~min}$. In these works, the color of the physical flower jelly depends on the food coloring that the user mixes into the colored jelly. To make multiple flowers in one base jelly, like in Figure 1 (C), users can move the bowl after each flower is printed so that multiple flowers can be centered at different locations in the same bowl. When making complex flowers that require multiple cutting knives like petals and stamens 
(Figure 8 (E), (F)), users can export multiple G-code files. In this case, users simply need to change the knives after each printing process is done.

\section{Mathematical Geometry}

In addition to flowers, our printer can also produce other 3D forms, as long as there are no overhangs in the design. For example, we can draw point-based images and messages in the jelly using the stamen needle by modifying the path planning algorithm (Figure $8(\mathrm{G})$ ). We can also create cubes using parallel slices in the jelly (Figure $8(\mathrm{H})$ ). Further research is required to explore the full variety of these non-floral shapes.

\section{USER STUDY}

\subsection{Overview}

We performed a user study to obtain feedback on our design software and the fabrication process. Our user study consisted of eight participants (three males and five females, age: 21 - 39 years old). Four participants had never made flower jellies before but had an experience of CAD / 3D printers, one participant had made flower jellies and used CAD / 3D printers several times, and the remaining three participants had extensive experience in creating flower jellies but never used CAD / 3D printers. We divided them into two groups, the novice group (the first four participants) and the experienced group (the latter four), and conducted different sessions for each group. Each individual was paid approximately $\$ 40$ for their participation. This study was approved by the ethical committee of The University of Tokyo.

To deal with the COVID-19 pandemic, we confirmed that the participants' body temperature was under $37.0^{\circ} \mathrm{C}$, the room was properly ventilated, and all the equipment was sanitized beforehand. We asked them to sanitize their hands with ethanol solution and wear masks throughout the user study. Also, to minimize the physical interaction, we skipped the manual fabrication process of flower jelly for the experienced group, since they had already known about it well.

\subsection{Process}

For each time slot of the study session, two participants gathered at the kitchen (i.e., four sessions in total). Each session was done as follows:

Novice group (four-hour session)

1. Manual fabrication

(1) They were instructed on the basics of what the flower jelly is and how to make one

(2) They manually fabricated a dahlia flower jelly $(6,9,11,17$, 17 petals for five different layers) with a syringe and the petal knife while looking at the actual photo of the flower

(3) They filled out a questionnaire on the experience of manual fabrication

2. Design and fabrication with Flower Jelly Printer

(1) They iterated on the design of a dahlia using the software and had the choice to use arbitrary numbers of petals and layers

(2) They exported the completed design and printed it
Table 1: Survey questionnaire.

$\begin{array}{ll}\text { Q1. [Appearance] } & \text { The appearance of the finished flower jelly is as you expected } \\ \text { Q2. [Difficulty] } & \text { Fabrication of the flower jelly is technically difficult for you } \\ \text { Q3. [Frustration] } & \text { You feel frustration when fabricating the flower jelly } \\ \text { Q4. [Enjoyability] } & \text { The fabrication process is enjoyable and fulfilling } \\ \text { Q5. [Individuality] } & \text { You feel that the finished flower jelly is individual to you }\end{array}$

Usability of Flower Jelly Printer

Q6. [Intuitive Interface] The software interface is easy to grasp

Q7. [Software Function] The software interface has needed functions for design

Q8. [Hardware Function] The printer has needed functions for design
User Experience

(3) They filled out a questionnaire on the experience of design and fabrication with Flower Jelly Printer

(4) We conducted a semi-structured interview on the answers to the questionnaire

Experienced group (two-hour session)

1. Manual fabrication (questionnaire only)

(1) They filled out a questionnaire on their typical experiences of manually fabricating flower jellies

2. Design and fabrication with Flower Jelly Printer

(1) They designed any type of flower without stamens in Figure 8 (A)-(D) using the software with arbitrary numbers of petals, layers, and colors

(2) They exported the completed design and printed it

(3) They filled out a questionnaire on the experience of design and fabrication with Flower Jelly Printer

(4) We conducted a semi-structured interview on the answers to the questionnaire

We allowed the experienced group to design with any type of flower without stamens and its color in the software in order to examine the large diversity of design choices might make. In this work, flowers using a stamen needle were not listed because they need too much time to print within the session. The questionnaire consisted of two sections (one on the user experience and one on the usability of Flower Jelly Printer) with 8 questions in total, as shown in Table 1. We asked the first five questions both for manual and printing fabrication, and asked the last three questions only for printing fabrication. The answers were rated on a 5-point scale (1. "No. not at all," 3. "Neither," 5. "Yes. very much") by participants.

\subsection{Results}

Figure 9 shows the mean scores of the two groups, (A) the novice group and (B) the experienced group, in response to the questionnaire in Table 1 ( $n=4$ for each graph, error bars mean standard errors).

For Q1 [Appearance], participants in the novice group reported increased satisfaction with the flower jelly's appearance (from 3.25 to 3.75) when using the Flower Jelly Printer versus manual fabrication. The experienced group responded that their satisfaction with the appearance of the printed flower was almost the same as with ones they made manually.

The scores on Q2 [Difficulty] show that the difficulty of making 


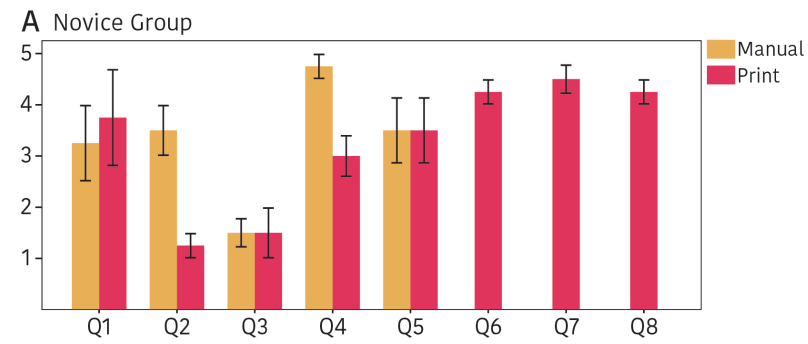

B Experienced Group

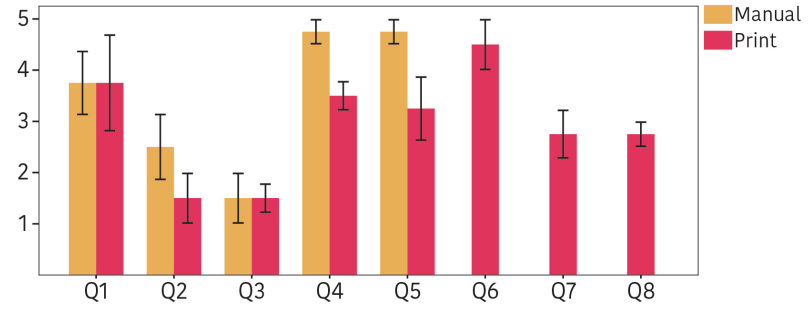

Figure 9: The mean scores of the questionnaire for the (A) novice and (B) experienced groups (error bar: standard error $\sigma / \sqrt{n}, n=4$ for each group)

flower jellies was decreased drastically by our proposed method in both groups, from 3.5 to 1.25 in the novice group and from 2.5 to 1.5 in the experienced group, respectively.

Looking at the result of Q4 [Enjoyability] and Q5 [Individuality], on the other hand, participants found the digital printing process to be less enjoyable and fulfilling. They also mentioned that the printed flower jelly seemed less unique to the creators.

In terms of Q6, Q7, and Q8 on the usability of the proposed system, novice users found it generally easy to understand and with enough useful features, giving scores over 4.0. However, while experienced users also scored the Flower Jelly Printer as easy to understand, they gave relatively lower scores for whether the software and printer provided the required functions for design, and gave numerous suggestions for improvements to our software and printer. These improvements are discussed in the Discussion and Future Work sections.

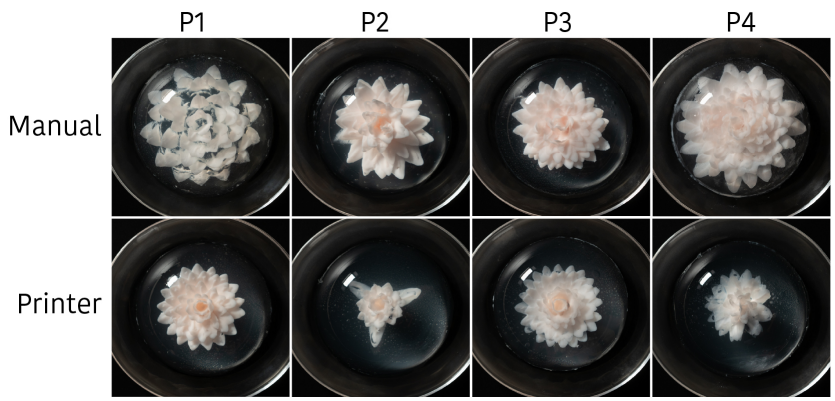

Figure 10: Dahlia patterns made with both manual (top) and printing processes (bottom) by the novice group.

\section{DISCUSSION}

Our results show that indeed the Flower Jelly Printer is easy to use and successfully enabled both novice and experienced users to create aesthetically pleasing flower jellies. However, users also shared some limitations and suggestions for improvement, especially in terms of the design functionalities and as a craft process.

The printer enabled easier and more precise fabrication, especially for novice users. According to P3, "Manually making flower jelly was difficult when I tried to distribute petals concentrically with equal distances," and P2 reported such challenges as "In case of manual fabrication, I needed to check the result from the bottom and the side of the jelly every time I made a new petal." The dahlia patterns made by the novice group in Figure 10, especially P1's photos, further reflect the positive change in the appearance going from manual to digital fabrication.

The digital design software also gave creators the benefit of being able to preview and iterate on designs before actually making physical jellies, enabling them to play more and create flowers that better matched their desired designs ( $\mathrm{P} 1$ : "The printed one was more precise because I could easily check the whole structure before starting the fabrication"). In the manual process, experienced users had to craft petal knives by hand cutting thin metal sheets and gluing needles to the blade. However, after creating the entire flower jelly, they often found the result of the handmade knife shape was different from what they imagined, and would need to remake the petal knife. As a result, the 3D preview was very helpful in saving both the time to iterate on petal knife shape and materials that would have otherwise been wasted on physical iteration. In fact, one experienced user found the design software helpful even without the printer as a way to test their designs (P7: "Trying many design patterns on the software was helpful. I think this is useful even when I make flower jellies manually").

However, with the convenience of digital fabrication, many users reported a decrease in the pleasure of the crafting process and resulting artifact. Part of this loss came from the printer taking on the work of the person. P4 shared that "In case of the manual process, I couldn't make a mistake, which was rather enjoyable when I finally accomplished it." It is possible that the printer made the task less risky and too easy, by removing the ability to make human mistakes. There was a less sense of accomplishment when the artifact was completed successfully by the machine.

Another experienced user also found that automation of the fabrication process reduced a sense of connection with the finished artifact. P8 reported that "Although it is me who designed the jelly, the printed one can be copied many times, which lost the feeling of the devotion to my artifact." The easier it is to replicate the object, the less personal the object becomes to the designer, especially if the designer's craft skill is removed from the fabrication process. This challenge may be a general problem of digital food fabrication, or in any instance where the machine takes over for the human hand. Making the automated process more enjoyable and special for the makers is a challenge we aim to address in our future work. 


\section{FUTURE WORK}

In addition to user responses to the existing design software and printer capabilities, we also received many suggestions from participants for new features, especially from the experienced group.

Regarding the design software, participants would like to be able to (1) tilt the angle of the whole flower to create more beautiful bouquets; (2) adding custom messages in text along with flowers; (3) arrange petals asymmetrically; and (4) print leaves and other objects. In the fabrication process, users responded that they would like (1) the ability to change ink jelly colors during the printing process and (2) the ability to change printing knife shapes easily during the printing process. While the current process allows users to use multiple cutting tools on one flower (like a stamen needle and petal knife), the process of manually switching out cutting tools is cumbersome for complex flowers that need large numbers of different cutting tools.

We would also like to enable more creative freedom by allowing users to directly sketch the shape of petal knives. Sketching functions makes it easier to design petals that have complex organic shapes like cherry blossom, Chinese peony, and cosmos. By opening up the knife shape design completely to users, we also hope it will allow and inspire users to create any shape inside the jelly, beyond the existing floral themes and text and broaden the genre of edible jelly foods.

Our digital design tool allows us not only to preview the visual outcome of the work but also to calculate the geometry of the finished form. We can apply this capability to give users additional fabrication information, such as being able to generate recipes specific to their design. For example, in the user study for the experienced group, we observed that people had to mix several batches of food coloring in efforts to successfully reproduce the color of the flower in the software. In future work, we would like to implement a software function that automatically computes the best mixing ratio of a few basic pigments for both novice and experienced users to more quickly achieve desired colors.

In addition to making one-off flower jellies, our proposed printing technique can be applied in a small batch production process. Today, there is no automated machine for producing flower jellies. Professionals seldom sell flower jellies because they require too much time and effort to produce, resulting in very few on market and at high prices for the few that are available. We may be able to tailor the machinery of our system for small batch production so that more people can enjoy flower jellies.

This work introduced the ability to experiment with soft textures or smooth mouthfeels using digital food fabrication. Currently, we use only one ink gel and one matrix gel. However, in future work we can experiment with different ink gels that have not only different colors but also different textures and flavors, allowing us to experiment with creating dynamic sensory experiences where the taste and feel of the food changes as people consume different parts of the cake.

Finally, our injection-based printing technique can also be applied

not only to making flower jellies but potentially in any other softtextured food. As potential design examples, here we categorize material, property, and application of slit injection printing, as shown in Figure 11. In terms of material, it will allow injecting fluidic materials like liquid, gel, and bubbles. In addition to gel, dough and porridge are considered to sustain the inner materials as outer materials. As for its property, the inner materials will affect the spatial variety of texture and flavor. As an application of such choices of materials and properties, for instance, we expect food for people with swallowing difficulty. In the case of normal meals, the meal tends to be too firm in spite of its rich texture and flavor. On the other hand, conventional pureed meals tend to suffer from poor texture and look. We foresee that the 3D printed food by slit injection printing will allow the meal that is tender enough yet also rich with its texture and look. We expect to inject lean (stiffer gel) and fat part (softer gel) as well as meat juice (liquid) inside to achieve rich texture compared to conventional pureed meals or printed food paste.

Material

Outer Material

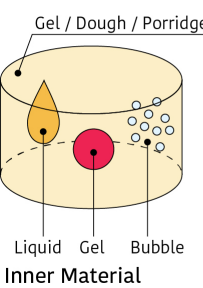

Inner Material
Property

Food by Slit Injection Printin

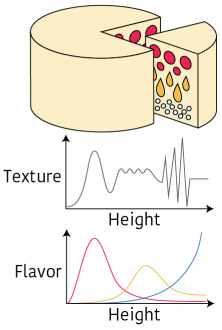

Figure 11: Potential design examples of slit injection printing based on its material, property, and application.

\section{CONCLUSION}

We presented the Flower Jelly Printer: a custom printing device and computational design tool for the design and digital fabrication of flower jellies. We implemented design software to allow users to play with design parameters and preview the resulting forms. Our newly developed slit injection printing technique directly injects colored jelly into a base jelly, which makes it possible to print of soft and fragile jelly structures. Our library of design examples demonstrates the aesthetic range of jellies that can be made with our system. The results of our user study showed that indeed our system improved the experiences of both novice and experienced creators by enabling trial-and-error design iteration and precise machine-augmented fabrication. We hope that more people will be able to enjoy creating and consuming these aesthetic desserts and that this work will open up new possibilities for personal design and fabrication of soft, gel-based foods.

\section{ACKNOWLEDGMENTS}

This work was supported by JST ERATO Grant Number JPMJER1501, Japan. The authors would also like to thank Dr. Jie Qi 
for the support in the writing process; and Prof. Yasuaki Kakehi and Prof. Takeo Igarashi for helpful comments.

\section{REFERENCES}

[1] byFlow. 2015. 3D Food Printer / Focus / byFlow. Retrieved September 17, 2020 from https://www.3dbyflow.com/

[2] Kentaro Fukuchi, Kazuhiro Jo, Akifumi Tomiyama, and Shunsuke Takao. 2012. Laser Cooking: A Novel Culinary Technique for Dry Heating Using a Laser Cutter and Vision Technology. In Proceedings of the ACM Multimedia 2012 Workshop on Multimedia for Cooking and Eating Activities (Nara, Japan) (CEA '12). Association for Computing Machinery, New York, NY, USA, 55-58. https://doi.org/10.1145/ 2390776.2390788

[3] Print2Taste GmbH. 2019. Mycusini. https://mycusini.com/.

[4] Kathleen Hajash, Bjorn Sparrman, Christophe Guberan, Jared Laucks, and Skylar Tibbits. 2017. Large-Scale Rapid Liquid Printing. 3D Printing and Additive Manufacturing 4, 3 (2017), 123-132. https://doi.org/10.1089/3dp.2017.0037

[5] Thomas J. Hinton, Andrew Hudson, Kira Pusch, Andrew Lee, and Adam W. Feinberg. 2016. 3D Printing PDMS Elastomer in a Hydrophilic Support Bath via Freeform Reversible Embedding. ACS Biomaterials Science \& Engineering 2, 10 (2016), 1781-1786. https://doi.org/10.1021/acsbiomaterials.6b00170 PMID 27747289.

[6] Thomas J. Hinton, Quentin Jallerat, Rachelle N. Palchesko, Joon Hyung Park, Martin S. Grodzicki, Hao-Jan Shue, Mohamed H. Ramadan, Andrew R. Hudson, and Adam W. Feinberg. 2015. Three-dimensional printing of complex biological structures by freeform reversible embedding of suspended hydrogels. Science Advances 1, 9 (2015). https://doi.org/10.1126/sciadv.1500758

[7] Rohit Ashok Khot, Deepti Aggarwal, Ryan Pennings, Larissa Hjorth, and Florian 'Floyd' Mueller. 2017. EdiPulse: Investigating a Playful Approach to SelfMonitoring through 3D Printed Chocolate Treats. In Proceedings of the $2017 \mathrm{CHI}$ Conference on Human Factors in Computing Systems (Denver, Colorado, USA) (CHI '17). Association for Computing Machinery, New York, NY, USA, 6593-6607. https://doi.org/10.1145/3025453.3025980

[8] Self-Assembly Lab. 2017. Rapid Liquid Printing - Self-Assembly Lab. Retrieved September 17, 2020 from https://selfassemblylab.mit.edu/rapid-liquid-printing

[9] Bokyung Lee, Jiwoo Hong, Jaeheung Surh, and Daniel Saakes. 2017. Ori-Mandu: Korean Dumpling into Whatever Shape You Want. In Proceedings of the $2017 \mathrm{CH}$ Conference Extended Abstracts on Human Factors in Computing Systems (Denver, Colorado, USA) (CHI EA '17). Association for Computing Machinery, New York, NY, USA, 456. https://doi.org/10.1145/3027063.3049777

[10] Ying-Ju Lin, Parinya Punpongsanon, Xin Wen, Daisuke Iwai, Kosuke Sato, Mar ianna Obrist, and Stefanie Mueller. 2020. FoodFab: Creating Food Perception Illusions Using Food 3D Printing. In Proceedings of the 2020 CHI Conference on Human Factors in Computing Systems (Honolulu, HI, USA) (CHI '20).
Association for Computing Machinery, New York, NY, USA, 1-13. https: //doi.org/10.1145/3313831.3376421

[11] Natural Machines. 2015. Natural Machines - Foodini. Retrieved September 17, 2020 from https://www.naturalmachines.com/foodini

[12] Moran Mizrahi, Amos Golan, Ariel Bezaleli Mizrahi, Rotem Gruber, Alexander Zoonder Lachnise, and Amit Zoran. 2016. Digital Gastronomy: Methods \& Recipes for Hybrid Cooking. In Proceedings of the 29th Annual Symposium on User Interface Software and Technology (Tokyo, Japan) (UIST '16). Association for Computing Machinery, New York, NY, USA, 541-552. https: //doi.org/10.1145/2984511.2984528

[13] Shigeru Owada. 2005. Fello Printer. Retrieved January 12, 2021 from https: //www2.sonycsl.co.jp/person/owd/JelloPrinter/

14] Ye Tao, Youngwook Do, Humphrey Yang, Yi-Chin Lee, Guanyun Wang, Catherine Mondoa, Jianxun Cui, Wen Wang, and Lining Yao. 2019. Morphlour: Personalized Flour-Based Morphing Food Induced by Dehydration or Hydration Method. In Proceedings of the 32nd Annual ACM Symposium on User Interface Software and Technology (New Orleans, LA, USA) (UIST '19). Association for Computing Machinery, New York, NY, USA, 329-340. https://doi.org/10.1145/3332165.3347949

[15] Wen Wang, Lining Yao, Teng Zhang, Chin-Yi Cheng, Daniel Levine, and Hiroshi Ishii. 2017. Transformative Appetite: Shape-Changing Food Transforms from 2D to 3D by Water Interaction through Cooking. In Proceedings of the $2017 \mathrm{CHI}$ Conference on Human Factors in Computing Systems (Denver, Colorado, USA) (CHI '17). Association for Computing Machinery, New York, NY, USA, 6123-6132. https://doi.org/10.1145/3025453.3026019

[16] Teresa Wegrzyn, Matt Golding, and Richard Archer. 2012. Food Layered Manufacture: A new process for constructing solid foods. Trends in Food Science \& Technology 27 (10 2012), 66-72. https://doi.org/10.1016/j.tifs.2012.04.006

[17] Wiiboox. 2015. Sweetin 3D Food Printer. https://www.wiiboox.com/3d-printerwiiboox-sweetin.php.

[18] Fan Yang, Min Zhang, and Bhesh Bhandari. 2015. Recent Development in 3D Food Printing. Critical reviews in food science and nutrition 57 (10 2015). https: //doi.org/10.1080/10408398.2015.1094732

[19] Amit Zoran. 2019. Cooking With Computers: The Vision of Digital Gastronomy. Proc. IEEE 107 (08 2019), 1467-1473. https://doi.org/10.1109/JPROC.2019.2925262

[20] Amit Zoran and Marcelo Coelho. 2011. Cornucopia: The Concept of Digital Gastronomy. Leonardo 44 (10 2011), 425-431. https://doi.org/10.2307/41421765

[21] Amit Zoran and Dror Cohen. 2018. Digital Konditorei: Programmable Taste Structures Using a Modular Mold. In Proceedings of the 2018 CHI Conference on Human Factors in Computing Systems (Montreal QC, Canada) (CHI '18). Association for Computing Machinery, New York, NY, USA, 1-9. https://doi.org/10. $1145 / 3173574.3173974$ 